\title{
Discussion
}

Lindblad: I have seen your results with great interest. It is interesting to note that they apply also to the Large Magellanic Cloud. Though this is a schematical presentation it can give a general orientation to the problem.

Perek: The purpose of this work was to obtain the relation between the general properties of orbits and the general features of various mass distributions. I have not attempted to obtain numerical values.

\section{COMPARISON OF THE MAGELLANIC CLOUDS WITH OTHER IRREGULAR BARRED SPIRALS}

\section{G. DE VAuCouleurs}

\section{University of Texas}

I. The main structural characteristics of the irregular barred spirals of the magellanic type are illustrated in Figure 1. They were first detected 10 years ago on small-scale photographs of the Large Cloud taken at Mount Stromlo (de Vau-

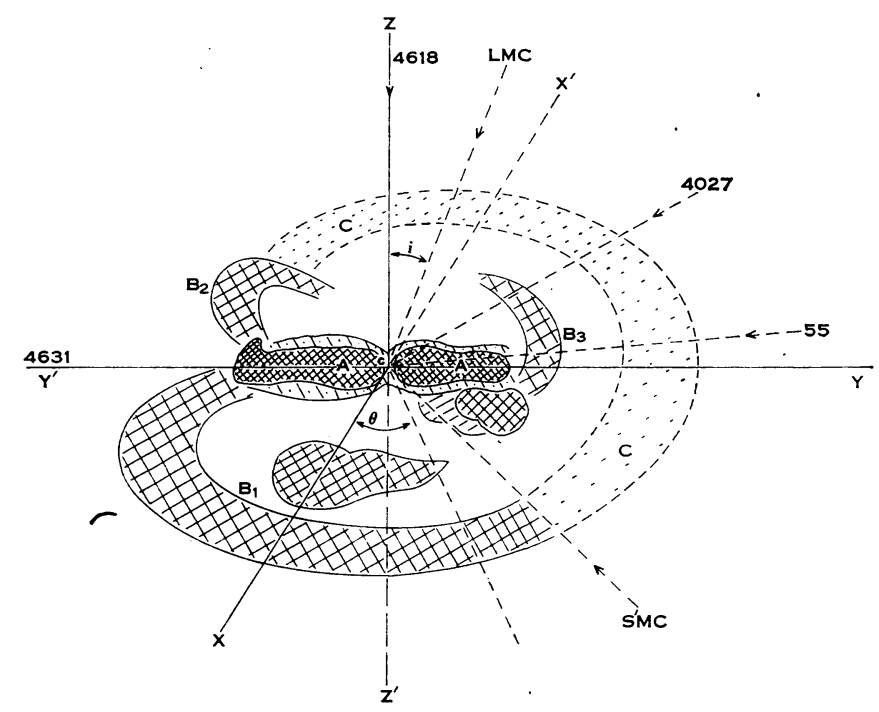

Fig. 1.-Structure of SB(s)m galaxies, and coordinate system.

couleurs 1954, 1955) and were later found to be uniformly present in many other "late-type" barred spirals designated $\mathrm{SB}(\mathrm{s}) \mathrm{d}$ and $\mathrm{SB}(\mathrm{s}) \mathrm{m}$ in the revised classification system (de Vaucouleurs 1959). These stages form a continuous transition between the regular barred spirals of the "S-shaped" sequences, such as NGC 1365: SB(s)b, NGC 1300: SB(s)bc, NGC 7479: SB(s)c, and the completely irregular system IBm showing only an axial bar and little or no traces of whorls, such as NGC 4449 and 4214. Examples of the transition stages are NGC 7741 : SB(s)cd, NGC 1313: SB(s)d, NGC 4027: SB(s)dm, NGC 4618: SB(rs)m, and the Large Magellanic Cloud: SB(s)m (Fig. 2). The Small Cloud, because of its interaction with the Large Cloud and its 

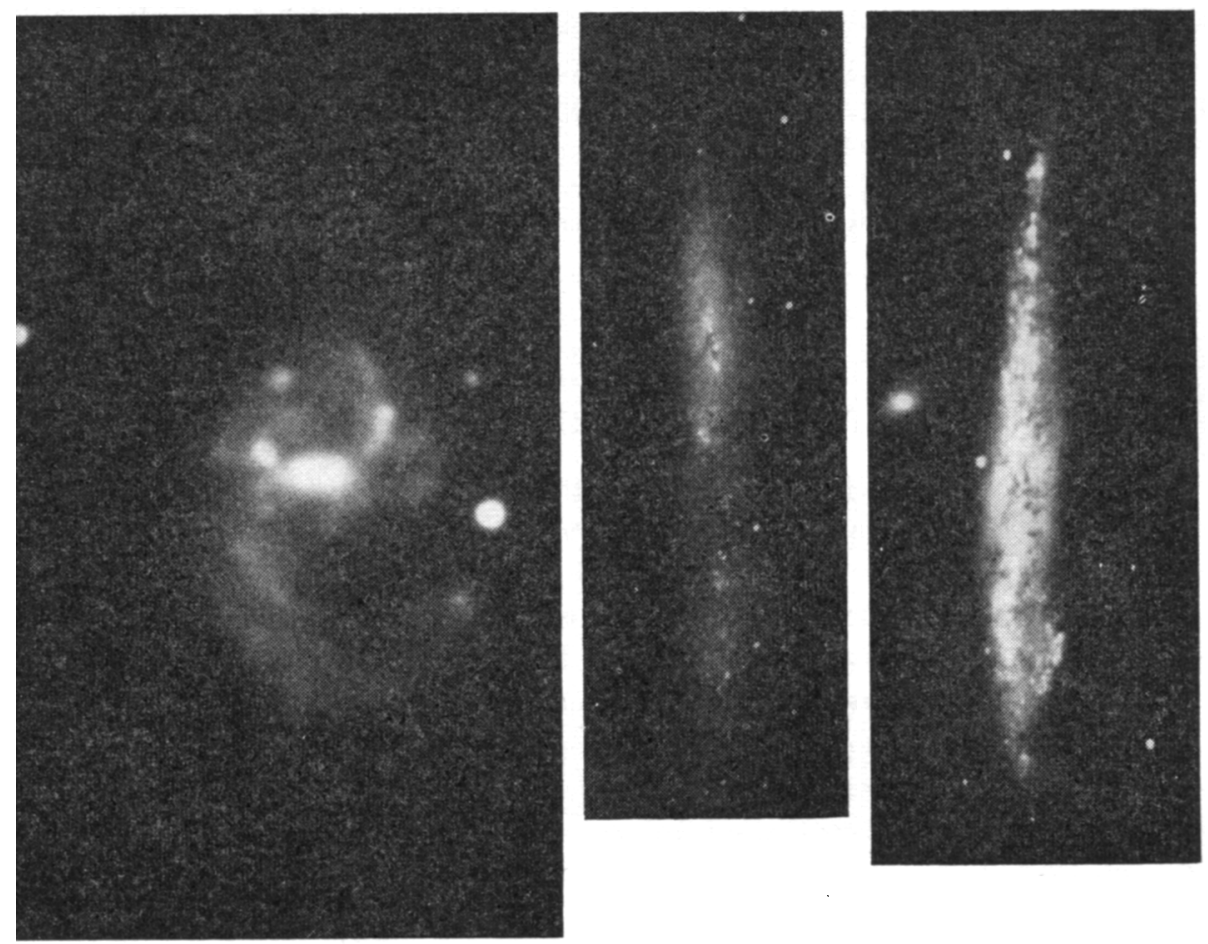

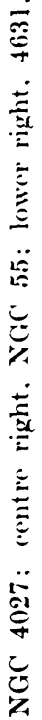

$\stackrel{\stackrel{ \pm}{x}}{\frac{x}{x}}$

$\stackrel{\check{c}}{\varrho}$
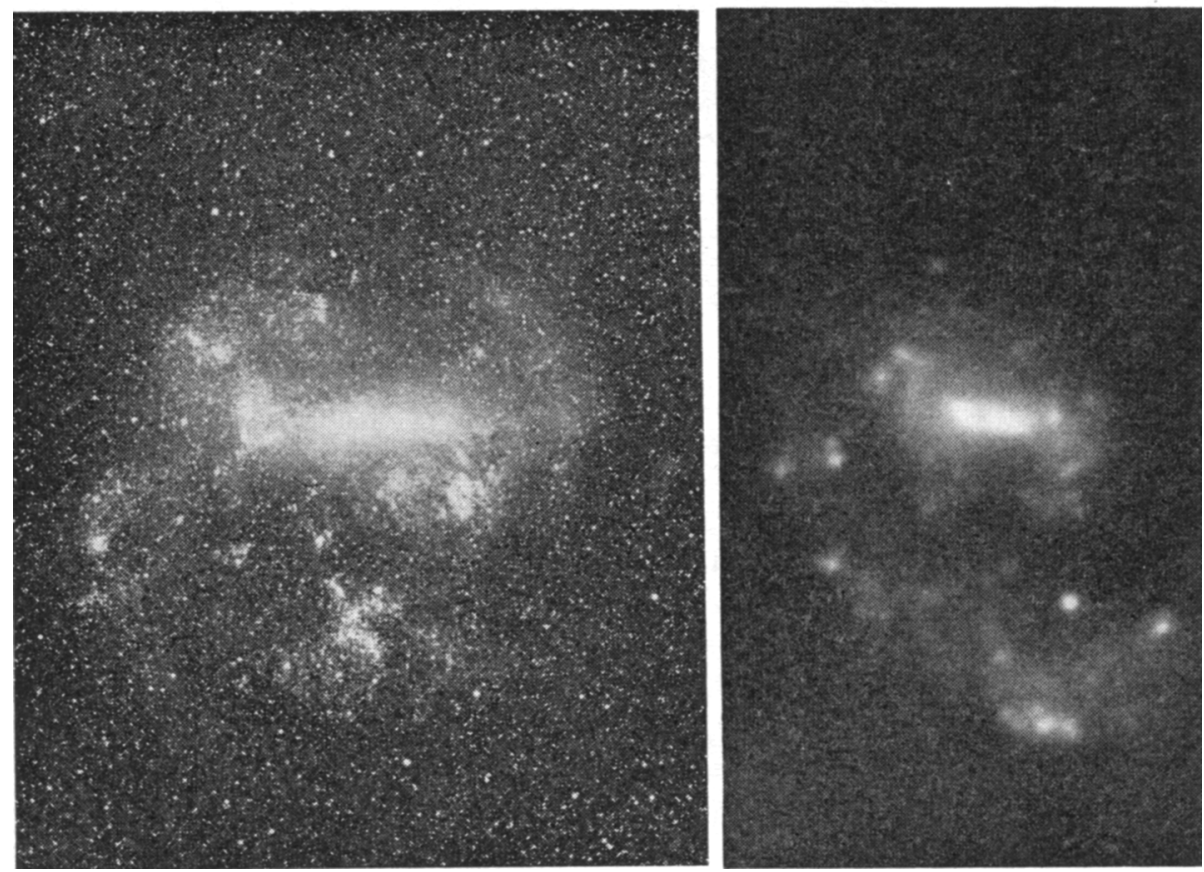

$\frac{\ddot{x}}{\ddot{t}}$

$\sum_{\nearrow}$

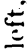

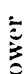

을

兰

起

'

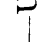

oi

sin 
unfavourable inclination, is more difficult to classify precisely; it may be designated as $\mathrm{SB}(\mathrm{s}) \mathrm{mp}$ or $\mathrm{IB}(\mathrm{s}) \mathrm{mp}$ to indicate the presence of residual (or incipient) spiral structure (de Vaucouleurs 1957a).

Three main regions may be recognized in magellanic-type systems (Fig. 1): region $\mathrm{A}$, the bar, is the brightest part, but by and large, it is poorer in OB supersupergiants than region $B$, including the main irregular and asymmetric spiral arm $\left(\mathrm{B}_{1}\right)$ and additional "embryonic" arms $\left(\mathrm{B}_{2}, \mathrm{~B}_{3}\right)$ where the richest $\mathrm{OB}$ associations and HII regions are found (de Vaucouleurs 1956); region C, a faint outer loop or whorl, is the extension of the main spiral arm encircling completely the central bar; this region lacks the bright supergiants and associated emission regions and is visible only on fully exposed photographs; its stellar population appears only past $m=15(M>-4)$ in the Magellanic Clouds and it seems to be very poor in neutral hydrogen.

A comparison of the optical brightness, star counts, radio continuum, and $21 \mathrm{~cm}$ emission in the Large Cloud was presented in 1955 at the Jodrell Bank Symposium (de Vaucouleurs 1957b) and the conclusions then reached remain valid. More detailed regional comparisons should now be possible thanks to the higher resolution of the new Australian radio telescopes.

II. The magellanic-type systems are "irregular" only by comparison with the more symmetrical text-book examples of barred spirals; it is essential, however, to realize that theirs is a very "regular" kind of irregularity, one which is faithfully reproduced in many other galaxies found in the same region of the classification volume. The main characteristic features and classification criteria are: $(a)$ the increasing asymmetry of the system, slight at SBc and large at SBm, (b) the decreasing visibility of the small nucleus in the centre of the bar, which is still bright at $\mathrm{SBc}$ and totally absent at SBm, and $(c)$ the degree of resolution and clumpiness of the arms, i.e. the abundance of $\mathrm{OB}$ associations - which as in the other spiral sequences increases from stage $\mathrm{SBc}$ to stage $\mathrm{SBm}$ and beyond. A detailed comparison of large reflector photographs of NGC 7741, 1313, 4027, 4618, etc., with small-scale photographs of the Magellanic Clouds will make these features fairly obvious.

III. Feature $(a)$ is probably the most remarkable and significant characteristic of the magellanic-type systems. This asymmetry is not only obvious in the spiral pattern, but it can be readily observed also in the surface distributions of (i) the resolved supergiant stars (de Vaucouleurs 1955), and correspondingly of HII regions (Henize and Miller 1951) and star clusters (Hodge 1960; also personal communication by B. Westerlund), (ii) the optical surface brightness, i.e. in effect the unresolved giant and main sequence stars contributing most of the luminosity (de Vaucouleurs 1957; G. and A. de Vaucouleurs 1959), (iii) the radio continuum emission (Mills 1955; Kerr 1962; see also paper 54 by D. S. Mathewson), and (iv) the neutral hydrogen emission (Kerr, Hindman, and Robinson 1954; Hindman, Kerr, and McGee 1963). All these distributions have centroids located approximately $1^{\circ}=0.8 \mathrm{kpc}$ to the north of the centre of symmatry of the bar in the Large Cloud. The same displacement and asymmetry is observed in other similar systems such as NGC 55, 1313, 4631 (de Vaucouleurs 1961, 1963; G. and A. de Vaucouleurs 1963) (see also paper 59 on NGC 55 by B. J. Robinson). 
IV. There are indications that this asymmetry may be reflected also in the velocity distribution. In 1954, Kerr and the present writer found that the $21-\mathrm{cm}$ line velocities would not lead to a symmetric rotation curve about the centre $C$ of the bar; in order to obtain a symmetric rotation curve amenable to standard analysis a fictitious "radio centre" $C_{\mathrm{r}}$ displaced about $1^{\circ}$ to the north of $C$ had to be introduced (Kerr and de Vaucouleurs 1955, 1956). This asymmetry may be also present in the stellar velocities of Feast, Thackeray, and Wesselink (1961).

Several hypotheses may be considered:

(a) The effect does not exist, i.e. the asymmetry is due to systematic observational errors in the $21-\mathrm{cm}$ line profiles observed in the low-intensity regions south of the bar; this hypothesis is not confirmed by more recent measurements with higher sensitivity and resolution (Hindman, Kerr, and McGee 1963).

(b) The effect is fictitious: this could be so if the tangential velocity component in the north-south direction were large enough to introduce significant line-of-sight components in regions several degrees away from the centre of mass; if so a velocity vector of the order $100 \mathrm{~km} / \mathrm{sec}$ in p.a. 330 to $360^{\circ}$ is required (Kerr and de Vaucouleurs 1955; Feast, Thackeray, and Wesselink 1961); this is an ad hoc hypothesis which there is at present no way of proving or disproving.

(c) The effect is real and, if so, either (i) the centre of mass of the system is really near $C_{\mathrm{r}}$, or (ii) large-scale departures from pure rotation are present. The systematic displacements of the centroids of the optical and radio distributions mentioned above tend to support hypothesis $(c)(\mathrm{i})$, but it is difficult to conceive how the centre of gravity of a barred spiral can be displaced by some $0.8 \mathrm{kpc}$ from the region of highest stellar density and from the centre of symmetry of the bar. The writer favours hypothesis $(c)$ (ii) which assumes only that large-scale streaming motions are present, at least in the interstellar gas (and possibly to some extent among the young supergiant stars which have recently condensed out of it). Recent spectroscopic observations of magellanic-type systems at McDonald Observatory seem to establish conclusively the reality and generality of such large-scale gas streaming in late-type barred spirals.

V. In order to interpret correctly the spectroscopic observations it is necessary to recognize the variable aspect of asymmetric magellanic-type systems depending on their orientation. In ordinary symmetric spirals the aspect of the system depends only, generally speaking, on the inclination of the rotation axis to the line of sight. In an asymmetric barred spiral the azimuth angle must be considered also.

In Figure 1 a coordinate system is attached to a magellanic-type spiral; the $X Y$ plane is the equatorial plane, the positive half of the $X$-axis is toward the stronger, asymmetric arm (the north side in the Large Cloud), the positive half of the $Y$-axis is toward the tip of the bar from which the weaker arm emerges (the east side in the Large Cloud), the positive half of the $Z$-axis given by the usual trigonometric convention is then such that the rotation of the system is retrograde (clockwise) with the main arm trailing. The direction of observation is defined by the inclination angle $i(=Z C O)$, and by the azimuthal angle $\theta\left(=X C O^{\prime}\right)$, if $C O^{\prime}$ is the projection of $C O$ on the $X Y$ plane. 
The presentation of the Large Magellanic Cloud corresponds to $i=27^{\circ}$ and $\theta=45^{\circ}$ (p.a. of the bar $=120^{\circ}$, p.a. of major axis $=165^{\circ}$ ). For the Small Cloud, $i \approx 60^{\circ}, \theta \approx 45^{\circ}$. For NGC 1313, $i \approx 40^{\circ}, \theta \approx 25$ or $205^{\circ}$ (de Vaucouleurs 1963). For NGC 4027, $i=39^{\circ}$, and for NGC 7741, $i=45^{\circ}, \theta \approx 90^{\circ}$, i.e. the bar coincides very nearly with the minor axis of the projected image (Fig. 2).

When a magellanic system is observed from a direction close to the equatorial plane its appearance depends very much on $\theta$, thus for NGC 55 (Fig. 3) $i=5^{\circ}$, $\theta \approx 90^{\circ}$, i.e. the bar is seen nearly end-on and from the "rear" (the positive direc-

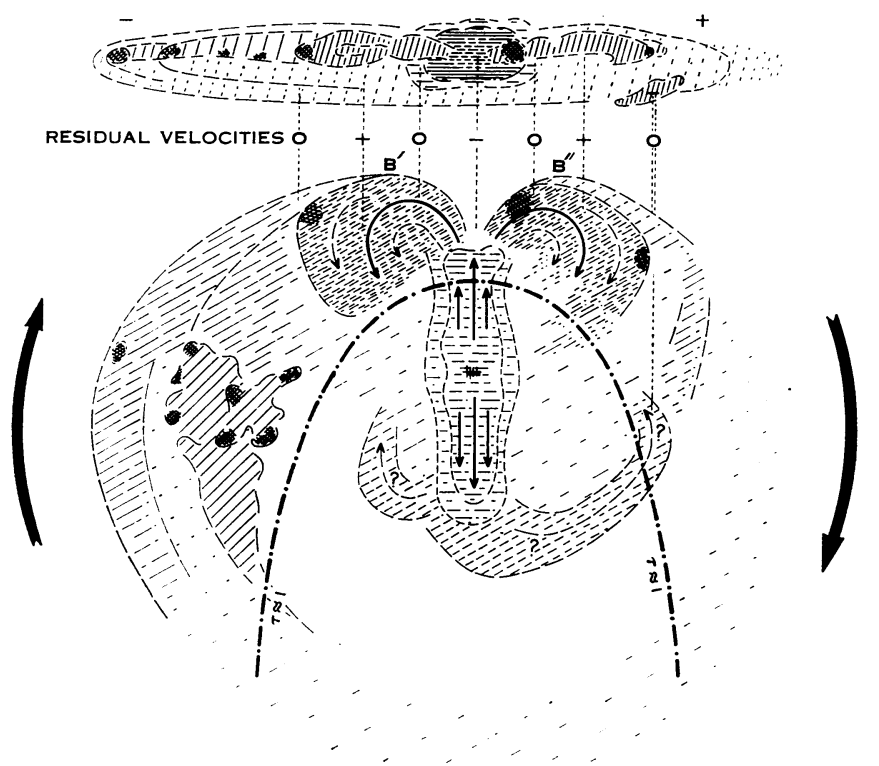

Fig. 3.-Gas streams in $\mathrm{SB}(\mathrm{s}) \mathrm{m}$ systems, and residual velocities in edgewise systems, e.g. NGC 4631.

tion on the $Y$-axis) (de Vaucouleurs 1961); for NGC 4631 (Fig. 3), $i=5^{\circ}, \theta=270^{\circ}$, i.e. the bar is seen end-on and from the "front" side (G. and A. de Vaucouleurs 1963). A three-dimensional model (displayed at meeting) is helpful to analyse the various appearances of magellanic-type systems seen from various directions. The basic structure of the Large Magellanic Cloud may be thus recognized in systems such as NGC 55, 2188, 2537, and 4027.

Once this structure is recognized, a logical interpretation of some "peculiar" or "distorted" velocity curves follows immediately. Thus the wavy velocity curve of NGC 4631 (G. and A. de Vaucouleurs 1963) could be interpreted in terms of the circulation pattern involving large-scale streaming motions of the gas in the bar (seen end-on) and eddy-like currents in the short "embryonic" arms as illustrated in Figure 3. In this galaxy, as in the Large Cloud, the rotation curve is symmetrical about a point displaced by some $0.8 \mathrm{kpc}$ from the bar toward the richer side of the system. 
VI. It may not be obvious to astronomers not familiar with the structure of the Magellanic Clouds that systems such as NGC 55 or NGC 4631 are really SB(s)m systems seen edgewise. In order to place the interpretation of the rotation curve and the reality of gas streaming in the bar beyond doubt, it was necessary to find typical SB(s)d and SB(s)m systems meeting the following conditions: $(a)$ the inclination angle is such that the spiral structure is immediately in evidence $\left(i<60^{\circ}\right)$, but not so small that (as may be the case of the Large Cloud) departures from circular motion will have negligible line-of-sight components, and $(b)$ the bar is in the $Y C O$ plane, i.e. is along the minor axis of the projected image $\left(\theta=90\right.$ or $\left.270^{\circ}\right)$ so that the rotational velocity will have no component on the line of sight and any line-shift

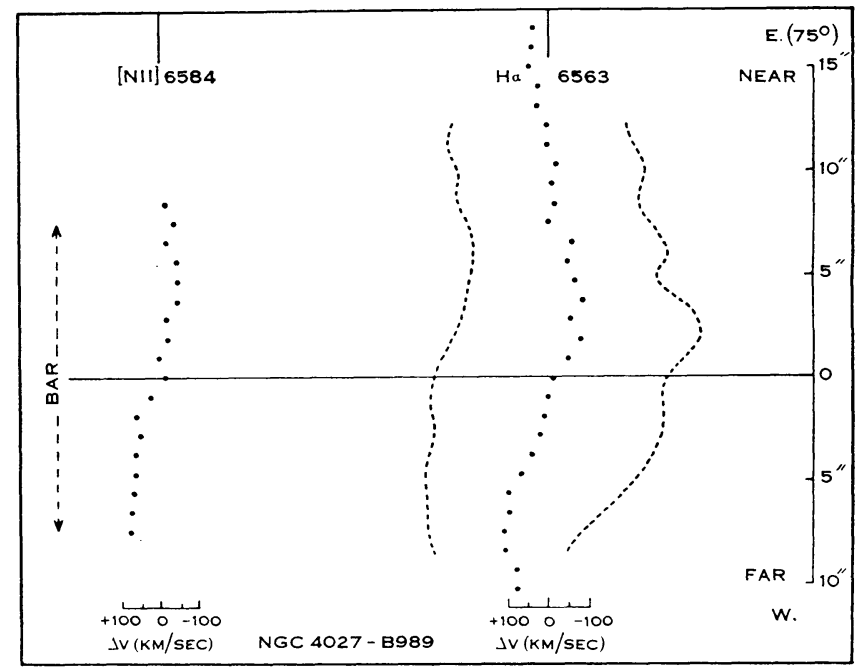

Fig. 4.- Shapes of $\lambda 6563(\mathrm{H} \alpha)$ and $\lambda 6584[\mathrm{NII}]$ in spectograms of bars of NGC 4027 and 7741, showing presence of gas stream. ing. (82-inch reflector, McDonald Observatory.)

observed will directly reflect departures from circular motion. Two systems meeting these rather strict requirements, NGC 4027 and NGC 7741, have been found and they were observed recently with the prime-focus spectrograph of the 82 -inch telescope at McDonald Observatory. Figure 4 shows the shapes of the $\lambda 6563(\mathrm{H} \alpha)$ and $\lambda 6584$ [NII] emission lines in spectrograms taken with the slit placed along the bars; in both galaxies the lines are conspicuously inclined and the geometric relationships are such that the tilt corresponds to stream velocities of the order of \pm 50 to $100 \mathrm{~km} / \mathrm{sec}$ directed along the bar and away from the centre. This direct evidence agrees well with the results previously derived from the analysis of NGC 4631, and to which the reader is referred for further details (G. and A. de Vaucouleurs 1963). A complete discussion of the rotation and stream motions in NGC 4027 and NGC 7741 will be published later after all the spectrograms taken in several position angles have been measured.

It would be especially interesting to detect similar stream motions in the Large Cloud through a suitable analysis of detailed observations of the $21-\mathrm{cm}$ emission 
with the 210-foot radio telescope. The presentation of the Large Cloud is, however, rather unfavourable because $(a)$ the bar $\left(\right.$ p.a. $=120^{\circ}$ ) is roughly at $45^{\circ}$ from the projected minor axis $\left(\right.$ p.a. $\left.=75^{\circ}\right)$, and $(b)$ the inclination $\left(i \approx 27^{\circ}\right)$ is small and difficult to estimate precisely. A stream velocity of the order of $V(s) \approx \pm 50 \mathrm{~km} / \mathrm{sec}$ would thus have a line-of-sight velocity component $V_{\mathrm{r}}(s) \approx \pm 15$ to $20 \mathrm{~km} / \mathrm{sec}$, which might be hard to disentangle from other components owing to a possible asymmetry of the velocity curve and to transverse motion.

The possibility of detecting more directly such motions in optical and perhaps 21-cm observations of other southern $\mathrm{SB}(\mathrm{s}) \mathrm{d}, \mathrm{SB}(\mathrm{s}) \mathrm{m}$ systems such as NGC 55, NGC 1313, NGC 2188, NGC 2442, and NGC 4945 should not be overlooked.

It is a pleasure to recall that the observations of the Magellanic Clouds at Mount Stromlo in 1952-54 were made possible through a Research Fellowship of the Australian National University and that many stimulating discussions of the radio observations were held with F. J. Kerr and B. Y. Mills. The current spectroscopic observations of magellanic-type galaxies at McDonald Observatory are supported by the U.S. Office of Naval Research and the University of Texas.

\section{References}

Feast, M. W., Thackeray, A. D., and Wesselink, A. J. (1961).-M.N. 122: 433-43.

Hexize, K. G., and Miller, F. D. (1951)._- "The Structure of the Galaxy: a Symposium." [Ann Arbor, Mich. 1950.] Publ. Obs. Univ. Mich. 10: 75-8.

Hindman, J. V., Kerr, F. J., and McGee, R. X. (1963).-Aust. J. Phys. 16: 570-83.

Hodge, P. W. (1960).-Ap. J. 131 : 351-7.

KERR, F. J. (1962).-Sky and Tel. 24 : 254-60.

Kerr, F. J., Hindman, J. V., and Robisson, B. J. (1954).-Aust. J. Phys. $7: 297-314$.

Kerr, F. J., and de VAucouleurs, G. (1955).-Aust. J. Phys. 8: 508-22.

Kerk, F. J., and de VAucouleurs, G. (1956).-Aust. J. Phys. 9: 90-111.

Mills, B. Y. (1955).-Aust. J. Phys. 8: 368-89.

DE VAUcouleurs, G. (1954).-Observatory $74: 23-31,157-64$.

de Vaucouleurs, G. (1955).-A.J. $60: 126-40,219-30$.

De Vaucouleurs, G. (1956).--Irish Astr. J. $4: 13-23$.

DE VAucouleurs, G. (1957a).-P.A.S.P. 69: $233-8$.

ie VAucolleurs, G. (1957b)._._"Radio Astronomy." (Ed. H. C. van de Hulst.) Symp. IAU 4: 244-50. [Jodrell Bank 1955.] (Cambridge Univ. Press.)

De Vaucouleurs, G. $(1957 c)$.-A.J. 62:69-82.

de Vaucouleurs, G. (1959)._- “Handbuch der Physik." (Ed. S. Flügge.) Vol. 53. pp. 275-310. (Springer-Verlag: Berlin.)

de Vaucouleurs, G. (1961).-Ap. J. 133: 405-12.

DE VAUCOUleurs, G. (1963).-Ap. J. 137: 720-32.

de Vaucouleurs, G., and de Vaucouleurs, A. (1959).-P.A.S.P. 71 : 83-91.

de Vaucouleurs, G., and de Vaucoulecrs, A. (1963).-Ap.J. 137: 363-75.

\section{Discussion}

Lindblad: I have seen with extreme interest the evidence for motion along the bar in barred galaxies and congratulate Dr. de Vaucouleurs on his result. I believe that an outward motion in the bar may be compensated by inflow of matter in what may be considered as inward extensions of the spiral arms aiming towards the central nucleus. A kind of circulation may occur here.

de Vaucouleurs: We seem to have direct evidence of some such circulations in NGC 4631, but we should be careful not to extend to early systems, i.e. SB(s)b or c such as NGC 1300 or 1365, results which have so far been obtained only in the later types SB(s)d and $\mathrm{m}$, such as NGC 
4027 and 7741. Also we need not consider a closed-circuit circulation pattern, as interstellar matter will be removed by star formation and presumably replenished by injection from the corona.

Shobbrook: Although there appears to be definite evidence for leading of these small arms, I believe I am correct in saying that there are no positive data indicating a leading motion of the large main arms in any of the spirals or irregulars.

de Vaucouleurs: The main arms of these magellanic irregulars are trailing, but if one calls the small, curved parts "arms", then one of them at least is definitely leading.

Feast: Do you believe that your model of the LMC is consistent with the observed rotation curve of gas and stars? There appears to be no evidence in these results of streaming along the bar.

de Vaucouleurs: There is no evidence because no one has yet analysed the velocity data with this possibility in mind. Radio astronomers, using high resolution, should look for gas streaming along the bar of the Large Cloud.

Kerr: There is no evidence in the radio observations so far for a streaming motion along the bar, but such a motion would be quite difficult to distinguish from error in the assumed galactic rotation velocity.

de Vaucouleurs: If we assume a streaming motion along the bar of $\pm 50 \mathrm{~km} / \mathrm{sec}$, the line-ofsight component should be only about \pm 15 to $20 \mathrm{~km} / \mathrm{sec}$, which certainly would be difficult to disentangle from other motions.

\section{COMPARISON OF NEUTRAL HYDROGEN IN NGC 55 AND THE LMC}

\section{B. J. Robinson and K. J. van Damme CSIRO Radiophysics Laboratory, Sydney}

Twenty-one-cm neutral hydrogen emission from NGC 55 has been measured with the 210-foot radio telescope at Parkes and a low-noise receiver (Robinson 1963). The beamwidth at $21 \mathrm{~cm}$ is 13.5. NGC 55 has been classified by de Vaucouleurs as a SB(s)m system similar to the LMC, seen edgewise and with the luminous bar nearly along the line of sight (de Vaucouleurs 1961). The outer isophote published by de Vaucouleurs has dimensions of 45 by $9^{\prime}$, centred about 2.'5 to the south-following side of the bright "nucleus" in the centre of the bar.

Hydrogen-line profiles measured at $8^{\prime}$ intervals along the major axis are shown in Figure 1. The displacement of the velocity of the profile peak clearly reveals the rotation of the system. The velocities of the peaks (relative to the Sun) have been plotted in Figure 2. The velocities agree moderately well with those determined for three emission objects by de Vaucouleurs (1961). The rotation curve shows a marked asymmetry about the centre of the bar, similar to that found previously for the LMC (Kerr and de Vaucouleurs 1955). The rotation in NGC 55 is symmetrical about a point on the major axis displaced $3^{\prime}$ on the south-following side of the "nucleus". Assuming a corrected distance modulus of $26 \cdot 3$ for NGC 55 (de Vaucouleurs 1961), the rotation centre lies $1.5 \mathrm{kpc}$ from the centre of the bar. The corresponding distance in the LMC is 800 pc.

The mass contained within a radius of $30^{\prime}$ from the centre of rotation is $2 \times 10^{10} M_{\odot}$, giving $M / L \simeq 5 \cdot 1$. The total mass will be somewhat greater than this. There will be a small contribution (perhaps 10\%) from matter lying more than 